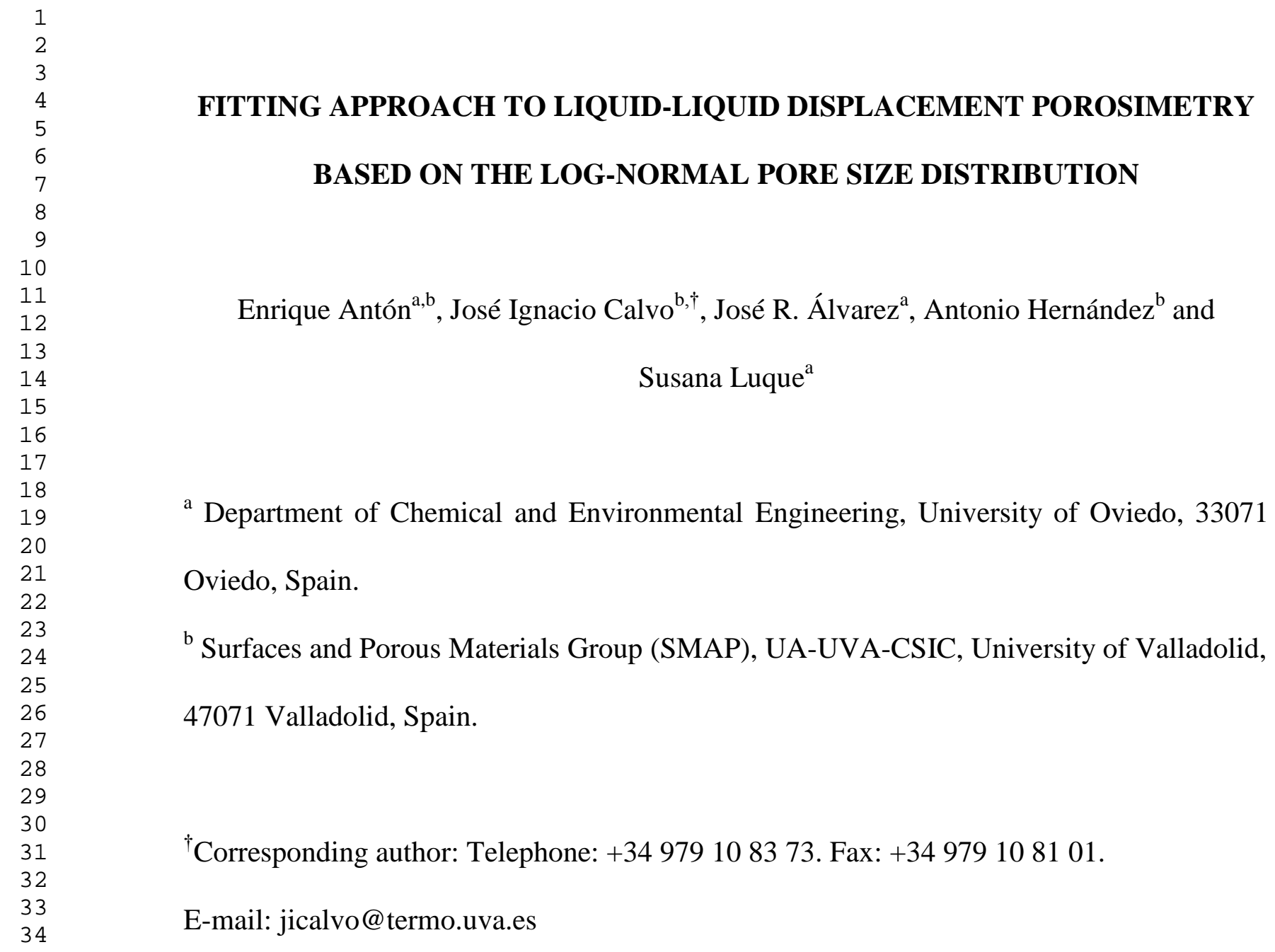

\section{FITTING APPROACH TO LIQUID-LIQUID DISPLACEMENT POROSIMETRY BASED ON THE LOG-NORMAL PORE SIZE DISTRIBUTION}

\author{
Enrique Antón ${ }^{\mathrm{a}, \mathrm{b}}$, José Ignacio Calvo ${ }^{\mathrm{b}, \uparrow}$, José R. Álvarez ${ }^{\mathrm{a}}$, Antonio Hernández ${ }^{\mathrm{b}}$ and \\ Susana Luque ${ }^{\mathrm{a}}$ \\ ${ }^{a}$ Department of Chemical and Environmental Engineering, University of Oviedo, 33071 \\ Oviedo, Spain. \\ ${ }^{\mathrm{b}}$ Surfaces and Porous Materials Group (SMAP), UA-UVA-CSIC, University of Valladolid, \\ 47071 Valladolid, Spain. \\ †Corresponding author: Telephone: +34 9791083 73. Fax: +34 979108101. \\ E-mail: jicalvo@termo.uva.es
}




\title{
Keywords
}

Membrane characterization

Ultrafiltration (UF) membranes

Liquid-liquid displacement porosimetry (LLDP)

Log-normal pore size distribution

Permeability distribution

\begin{abstract}
Liquid-liquid displacement porosimetry (LLDP) has been used to characterize several UF membranes in a wide range of molecular weight cut-offs (MWCO). A new method to convert porosimetric data into pore size distributions and related information has been developed based on assuming log-normal pore size distributions. The results of this are in good agreement with those from the customary data conversion algorithm (as derived by Grabar and Nikitine). The proposed method can also be used when a reduced number of experimental data points is available, leading to a significant reduction of data acquisition time needed to complete a reliable analysis.
\end{abstract}




\section{Introduction}

Membranes and membrane processes are regularly used in industrial applications for both gas and liquid separations. These applications cover from separations of particles and macromolecules to small ions, and the characteristics of the employed filters vary accordingly. For most of the separations based on the application of a pressure gradient across the membrane, namely microfiltration (MF), ultrafiltration (UF) and nanofiltration (NF), sieving is the main separation mechanism and therefore, the relative size of the membrane pores/interstices and the molecules to be retained, is the key factor to control separation. The typical membrane structure consists of a separation controlling layer (active layer), supported on a more open pore substructure (support layer) intended to give mechanical stability and resistance to the resulting membrane.

A proper knowledge of the porous structure of a membrane is very important to assess its separation capabilities.

This kind of knowledge is the target of multiple characterization methods than can be grouped under the term porometries. These methods are based on very different physical principles but all of them try to obtain about the pore size distribution (PSD), from which important separation parameters such as mean, maximum and minimum pore sizes, porosity or pore density can be calculated. Methods based on the bubble point test have been gaining recognition due to their unique capabilities. For example these methods test the membrane in wet state, very close to the real operation conditions. In addition, the information given refers only to active layer pores (even when the support is not detached from the whole membrane).

There are two methods based on the bubble point: the gas-liquid displacement porosimetry (GLDP) and the liquid-liquid displacement porosimetry (LLDP), whose main difference 
relies on the state of the fluids used for displacing the inner liquid. Both techniques have been indistinctly named as capillary flow porometry, [1], liquid extrusion porometry, [2], or even combined bubble pressure and solvent permeability method, [3, 4], but all of them refer to the same principle, [5].

Both LLDP and GLDP are well-known and very similar in concept and even in operation mode. Nevertheless GLDP has gained general recognition while LLDP is still scarcely used, because it is more difficult to operate and less reproducible. Some of the authors have been working over in the last years to improve LLDP in an effort to show the potential of the method, especially for tight UF and NF membranes where other methods have strong difficulties to get reliable results. One of the features of the LLDP that makes it less attractive than GLDP is the shape of the distributions it provides. Certainly the nice aspect of the GLDP results is a consequence of a continuous measurement procedure. Commercial GLDP apparatuses usually divide the experimental range in 256 data points and determine corresponding data pairs (flux, pressure), resulting in a very smooth Gaussian distribution. The same procedure is not accomplished in LLDP because liquid-liquid equilibrium usually takes longer time (the whole experiment should take some hours) and there is no guarantee of obtaining superior results to GLDP.

Different algorithms are used to process data from GLDP and LLDP experiments. Gasliquid experiments need to account for different gas flow regimes, namely Knudsen molecular flow along with Hagen-Poiseuille convective transport [6]. Moreover, the dependence of the gas permeability with applied pressure requires a different experimental procedure (wet run followed by dry ones) and different algorithms to convert experimental results into pore size information. The algorithms which could be used for processing data from LLDP experiments have been reviewed by Morison [7], who found that all of them 
are very sensitive to experimental errors requiring some smoothing to get reasonable distributions. Some authors have performed spline smoothing to get better results from LLDP, $[8,9]$, based on a polynomial fitting that somehow loses the physical meaning.

The approach in this work is to begin assuming a log-normal PSD and then fit the experimental results to such theoretical function. A similar approach was used by Aimar et al. [10] to fit log-normal distributions from retention data, sometimes combined with moment theory to get more insight into theoretical distributions [11, 12]. Most of the membranes found in the market are well described by a log-normal distribution of pore sizes, [13], with a continuous range from many very small pores to few much bigger ones. This should lead to a right skewed distribution which is better described by a log-normal function.

\section{LLDP theory}

\subsection{Traditional methods to determine pore number distribution}

The final aim of LLDP characterizations is to determine the PSD of a porous sample, in this case a membrane. This technique is based on the Young-Laplace equation which relates the surface tension of a fluid inside a capillary with the radius of such capillary. The experimental procedure consists in forcing a liquid to enter the pores of a membrane previously filled with another immiscible liquid (wetting liquid).

If a perfect wetting of the membrane by the wetting fluid is assumed, the needed pressure to empty a given cylindrical pore is related to the radius of such pore through the so called Cantor equation (Eq. (1)) [14].

$$
\Delta \mathrm{P}=\frac{2 \gamma}{\mathrm{r}}
$$


where $\Delta \mathrm{P}$ is the applied pressure and $\gamma$ the interfacial tension $(\mathrm{N} / \mathrm{m})$ between both liquids and $\mathrm{r}$ the equivalent pore radius.

This technique accounts for the narrowest section of the pores, because these pore-throats effectively govern the fluid transport and the retention capabilities of the membrane, no matter how complicated the membrane structure is.

The increase in the applied pressure is linked to an increase in the flow due to the opening of new smaller pores. Therefore, by measuring the equilibrium pressure drop corresponding to each increment of flux, the basic experimental information from LLDP is obtained.

A transport model inside the pores is then required to get the PSD. The Hagen-Poiseuille equation through capillary cylindrical pores is regularly used for convective transport of liquids inside pores. This geometry assumption is not as restrictive as it may look, since many membrane geometries can be simplified to a group of more or less straight cylindrical t pores having a radius equal to the narrowest section of the actual pores found in the membrane structure. Therefore, the flux $J_{i}\left(\mathrm{~m}^{3} / \mathrm{m}^{2} \mathrm{~s}\right)$ associated to the pores of radius $r_{i}(m)$ of the membrane, when a transmembrane pressure $\Delta \mathrm{P}(\mathrm{Pa})$ is applied, is given by the Hagen-Poiseuille equation (Eq. (2)).

$\mathrm{J}_{\mathrm{i}}=\frac{\mathrm{N}_{\mathrm{i}} \pi \mathrm{r}_{\mathrm{i}}^{4}}{8 \eta \mathrm{l}} \Delta \mathrm{P}$

where $\mathrm{N}_{\mathrm{i}}\left(\right.$ pore $\left./ \mathrm{m}^{2}\right)$ is the pore number density of pores having a radius $r_{i}, \eta(\mathrm{Pa} \cdot \mathrm{s})$ is the viscosity of the displacing liquid and $1(\mathrm{~m})$ is the length of the pores (usually the active layer thickness).This term should include a tortuosity factor for not so regular geometries However, the experimental flow values obtained are not associated to a single pore size, but to those pores with radii higher or equal to the radius obtained through the Cantor equation (Eq. (1)) for the given applied pressure. Thus, Eq. (2) cannot be directly applied to obtain 
the number of pores of a given pore size and then, more complex mathematical procedures have to be carried out in order to discriminate the contribution of each pore size to the global flux. Different methods have been developed for that purpose, such as the original method of Erbe [15], based on a graphical evaluation, and the method of Grabar and Nikitine [14], which has been selected for this work, and it will be briefly explained below The volumetric flux for a given $\Delta \mathrm{P}(\mathrm{J}(\Delta \mathrm{P}))$ is defined in terms of the number of pores per unit area through Eq. (3).

$J(\Delta P)=N \int_{r}^{r_{\max }} J_{V}(r, \Delta P) f_{n}(r) d r$

where $\mathrm{N}$ is the total number of pores per unit area $\left(\right.$ pore $\left./ \mathrm{m}^{2}\right), \mathrm{J}_{\mathrm{V}}(\mathrm{r}, \Delta \mathrm{P})$ is the volumetric flow $\left(\mathrm{m}^{3} / \mathrm{s}\right)$ through a single pore of radius $r$ at $\Delta P$ and $f_{n}(r)$ is the probability distribution function value for a pore of radius $r$.

The pore number distribution $(\mathrm{n}(\mathrm{r}))$ is defined as the number of pores per unit area and per unit radius, and can be calculated using Eq. (4) which is based on the probability distribution function $\mathrm{f}_{\mathrm{n}}(\mathrm{r})$.

$\mathrm{n}(\mathrm{r})=\mathrm{Nf} \mathrm{f}_{\mathrm{n}}(\mathrm{r})$

Therefore, the number of pores per unit area with radii between $r_{A}$ and $r_{B}\left(N_{A B}\right)$ is given by Eq. (5). Note that if the limits of integration are 0 and $\infty$ the result of the integral is the total pore population, $\mathrm{N}$.

$\mathrm{N}_{\mathrm{AB}}=\int_{\mathrm{r}_{\mathrm{A}}}^{\mathrm{r}_{\mathrm{B}}} \mathrm{n}(\mathrm{r}) \mathrm{dr}=\int_{\mathrm{r}_{\mathrm{A}}}^{\mathrm{r}_{\mathrm{B}}} \mathrm{Nf}_{\mathrm{n}}(\mathrm{r}) \mathrm{dr}$

Taking into account the Hagen-Poiseuille equation and Eq. (4), it is possible to rewrite Eq. (3) to obtain the volumetric flux, as long as the variables are assumed to be independent of pressure. 


$$
\mathrm{J}(\Delta \mathrm{P})=\frac{\pi \Delta \mathrm{P}}{8 \eta 1} \int_{\mathrm{r}}^{\infty} \mathrm{r}^{4} \mathrm{n}(\mathrm{r}) \mathrm{dr}
$$

where the limits of integration are the lowest radius which is opened at the applied transmembrane pressure $\Delta \mathrm{P}$ (given by Cantor equation) and the highest radius of the membrane which is denoted as $\infty$, because probability distribution is 0 for $r>r_{\max }$.

According to Grabar and Nikitine method [13], Eq. (6) has to be differentiated, substitute radius by pressure using the Cantor equation, and then, calculate the number of pores per unit area and per unit radius for a given differential of pressure through Eq. (7).

$$
\mathrm{n}(\mathrm{r})=\frac{8 \eta 1 \Delta \mathrm{P}^{5}}{\pi(2 \gamma)^{5}}\left[\frac{\mathrm{dJ}}{\mathrm{d}(\Delta \mathrm{P})}-\frac{\mathrm{J}}{\Delta \mathrm{P}}\right]
$$

The algorithm derived by Grabar and Nikitine is, essentially, a differential algorithm which requires the continuous curve of permeability variation for its derivation. However, experimental procedures only give discrete values of flow and pressure, so Eq. (7) has to be converted in an incremental equation as follows.

$$
\mathrm{n}\left(\mathrm{r}_{\mathrm{av}}\right)=\frac{8 \eta 1 \Delta \mathrm{P}_{\mathrm{av}}^{5}}{\pi(2 \gamma)^{5}}\left[\frac{\Delta \mathrm{J}}{\Delta(\Delta \mathrm{P})}-\frac{\mathrm{J}_{\mathrm{av}}}{\Delta \mathrm{P}_{\mathrm{av}}}\right]
$$

where the subscript "av" indicates the average value of the variables in the given increment and $\Delta \mathrm{J}$ and $\Delta(\Delta \mathrm{P})$ are the increments between two experimental consecutive data pairs, $\Delta \mathrm{J}=\mathrm{J}_{\mathrm{i}}-\mathrm{J}_{\mathrm{i}-1}$ and $\Delta(\Delta \mathrm{P})=\Delta \mathrm{P}_{\mathrm{i}}-\Delta \mathrm{P}_{\mathrm{i}-1}$, where the subscript $\mathrm{i}$ is the $\mathrm{i}$-th experimental point. The ratio $\Delta \mathrm{J} / \Delta(\Delta \mathrm{P})$ is the permeability increment $(\Delta \mathrm{L})$ while the ratio $\mathrm{J}_{\mathrm{av}} / \Delta \mathrm{P}_{\mathrm{av}}$ is the mean permeability $\left(\mathrm{L}_{\mathrm{av}}\right)$ in that increment. These ratios represent the slope of the porosimetric curve in the considered point and the slope of the straight line passing from that point and the origin, respectively. 
In conclusion, from the experimental flow values as a function of pressure and knowing the membrane area and thickness, Eq. (8) can be used to get the number of pores per unit area and unit radius $(\mathrm{n}(\mathrm{r}))$. From this pore number distribution, it is possible to determine several membrane parameters as explained in section 2.3.

\subsection{New approach to analyze LLDP results}

In our new approach, the idea is to create a theoretical membrane with a log-normal pore number distribution and total pore number density that produces the actual membrane flux as function of pressure.

The first step is to define a suitable probability distribution function $\left(f_{n}(r)\right)$ for the theoretical membrane. As mentioned above, among the several probability density functions, it has been reported that log-normal distribution is suitable for the pore number distribution of many membranes [7, 16]. The log-normal distribution is given by Eq. (9).

$$
\mathrm{f}_{\mathrm{n}}(\mathrm{r})=\frac{1}{\ln (\mathrm{S}) \mathrm{r} \sqrt{2 \pi}} \exp \left[-\frac{1}{2}\left(\frac{\ln (\mathrm{r} / \mathrm{R})}{\ln (\mathrm{S})}\right)^{2}\right]
$$

where the parameters $\mathrm{R}(\mathrm{m})$ and $\mathrm{S}$ (dimensionless) are the geometric mean radius or location parameter and the geometric standard deviation or scale parameter, respectively. Therefore, the pore number distribution of the membrane can be obtained by combining Eqs. (4) and (9).

$$
\mathrm{n}(\mathrm{r})=\frac{\mathrm{N}}{\ln (\mathrm{S}) \mathrm{r} \sqrt{2 \pi}} \exp \left[-\frac{1}{2}\left(\frac{\ln (\mathrm{r} / \mathrm{R})}{\ln (\mathrm{S})}\right)^{2}\right]
$$

where $\mathrm{N}$ is the total pore number density $\left(\right.$ pore $/ \mathrm{m}^{2}$ ) which is the third and last parameter of this model. 
Using Eq. (6), which gives the flux for a given transmembrane pressure $\Delta \mathrm{P}$, the membrane surface area (A) and the pore number distribution given by Eq. (10), the flow at a given pressure is obtained as follows.

$$
\mathrm{J}(\Delta \mathrm{P})=\frac{\mathrm{NA} \pi \Delta \mathrm{P}}{8 \sqrt{2 \pi} \ln (\mathrm{S}) \eta \mathrm{l}} \int_{\mathrm{r}}^{\infty} \mathrm{r}^{3} \exp \left[-\frac{1}{2}\left(\frac{\ln (\mathrm{r} / \mathrm{R})}{\ln (\mathrm{S})}\right)^{2}\right] \mathrm{dr}
$$

The limits of integration are again the radius of the smallest pore which is opened at the applied transmembrane pressure $\Delta \mathrm{P}$ and the radius of the biggest one.

The next task is to fit N, R and S to the LLDP characterization values in order to make this theoretical membrane behaves as closely as possible to the real one

For that purpose initial values are given to the $\mathrm{R}, \mathrm{S}$ and $\mathrm{N}$ parameters. This step is of great importance in real applications (such as in quality control processes), since the fitting procedure strongly depends on how close are these values to the final ones. Nevertheless, as the goal of this work is to evaluate the feasibility of the proposed approach, the initial values of $\mathrm{R}, \mathrm{S}$ and $\mathrm{N}$ were $1.5 \cdot 10^{-9} \mathrm{~m}, 1.5$ and $5 \cdot 10^{15}$ pore $/ \mathrm{m}^{2}$, respectively, for all the membranes and procedures (estimations based on previous knowledge about such values for typical membranes).

LLDP characterizations give discrete pairs of flow and pressure values. Given the initial values of $\mathrm{R}, \mathrm{S}$ and $\mathrm{N}$ and using the experimental pressure values, in Eq. (11), a first estimate of the flow can be obtained. Usually, calculated flow values are far from the experimental ones and new $\mathrm{R}, \mathrm{S}$ and $\mathrm{N}$ values have to be found. For this, an objective function was created using the sum of the squared residuals of the k-th flow data, defined as the square of the difference between the experimental flow $\left(\mathrm{J}_{\mathrm{k}, \text { experimental }}\right)$ and the flow provided by the model $\left(\mathrm{J}_{\mathrm{k}, \text { modelled }}\right)$ as follows. 
$\mathrm{e}_{\mathrm{k}}^{2}=\left(\mathrm{J}_{\mathrm{k}, \text { experimenal }}-\mathrm{J}_{\mathrm{k}, \text { modelled }}\right)^{2}$

The objective function is minimized by the least squares method (using the GRG2 algorithm as implemented in Microsoft Solver). In this way, the fitted parameters R, S and $\mathrm{N}$ correspond to a theoretical membrane whose behavior resembles that of the actual membrane.

One of the advantages of this model is that no differentiation of Eq. (3) is needed and thus, errors are minimized. In addition, the discrete experimental information is transformed into a continuous function, allowing more comprehensive analysis of the resulting distributions, which can be compared from the parameters of the resulting log-normal PSD's.

\subsection{Membrane properties from LLDP analysis}

The LLDP results give a pore number distribution as a function of the radius $(\mathrm{n}(\mathrm{r}))$, from where, it is possible to determine several parameters:

1) The differential pore number distribution, expressed as a percentage, is given by Eq. (13).

Differential pore number distribution $(\%)=\frac{\mathrm{n}(\mathrm{r})}{\int_{0}^{\infty} \mathrm{n}(\mathrm{r}) \mathrm{dr}} 100=\frac{\mathrm{n}(\mathrm{r})}{\mathrm{N}} 100$

2) In order to determine the permeability distribution of the membranes, the probability distribution function $\mathrm{f}_{\mathrm{L}}(\mathrm{r})\left(\mathrm{m}^{-1}\right)$ has to be defined to calculate the permeability $L_{A B}$ associated to the pores with radius between $r_{A}$ and $r_{B}$ with respect to the total permeability $\left(\mathrm{L}_{\mathrm{total}}\right)$.

$$
\frac{\mathrm{L}_{\mathrm{AB}}}{\mathrm{L}_{\text {total }}}=\int_{\mathrm{r}_{\mathrm{A}}}^{\mathrm{r}_{\mathrm{B}}} \mathrm{f}_{\mathrm{L}}(\mathrm{r}) \mathrm{dr}
$$


Taking into account that $\mathrm{L}=\mathrm{F} / \Delta \mathrm{P}$, Eq. (6) can be rewritten, so the integration from $r_{A}$ to $r_{B}$ and from 0 to $\infty$ give $L_{A B}$ and $L_{\text {total }}$, respectively. By combining all the equations, the probability distribution function for the permeability can be obtained as shown in Eq. (15).

$$
f_{L}(r)=\frac{r^{4} f_{n}(r)}{\int_{0}^{\infty} r^{4} f_{n}(r) d r}
$$

Therefore, the differential permeability distribution, expressed as percentage, is given by Eq. (16).

Differential permeability distribution $(\%)=\frac{\mathrm{f}_{\mathrm{L}}(\mathrm{r})}{\int_{0}^{\infty} \mathrm{f}_{\mathrm{L}}(\mathrm{r}) \mathrm{dr}} 100$

3) Finally the cumulative distributions, expressed as percentage, are the summation of the differential distributions from radius zero to radius $r$.

4) The asymptotic permeability is the permeability once all the pores have been opened and it was already denoted as $\mathrm{L}_{\text {total }}$.

5) The mean radius based on the pore number distribution $\left(\langle r\rangle_{\text {number }}\right)$ gives the mean pore radius of the membrane weighting the radius of each pore by the number of them (Eq. (17)).

$$
<\mathrm{r}>_{\text {number }}=\frac{\sum_{\forall \mathrm{i}} \mathrm{n}\left(\mathrm{r}_{\mathrm{i}}\right) \mathrm{r}_{\mathrm{i}}}{\sum_{\forall \mathrm{i}} \mathrm{n}\left(\mathrm{r}_{\mathrm{i}}\right)}
$$

6) The mean radius based on the permeability distribution $\left.(<r\rangle_{\text {perm }}\right)$ gives the mean pore radius of the membrane weighting the radius of each pore class by the permeability related to them (Eq. (18)). 


\section{Experimental}

\subsection{Membranes and sample preparation}

A variety of commercial flat-sheet membranes with MWCO ranging from 5 to $300 \mathrm{kDa}$ were characterized by LLDP and the explained technique. The properties of these membranes, according to manufacturers, are gathered in Table 1.

In order to applied LLDP analysis, several membrane coupons were cut in flat disk pieces where the effective diameter for the analysis was $36 \mathrm{~mm}$, as defined by the holder size. All the analyses were done to the pristine membranes which were conditioned by washing and soaking them in Milli-Q water for at least $48 \mathrm{~h}$. Then, previously to the characterization, membranes were soaked in the wetting liquid for $12 \mathrm{~h}$. Just before the analysis, the soaking was carried out under vacuum (created by a water jet pump) for 45 min to ensure the complete wetting of the sample.

\section{Table 1}

\subsection{LLDP porosimeter and analysis procedure}


The LLDP porosimeter was set up and tested at the University of Valladolid [19-21]. Both the equipment and the experimental procedure have been comprehensively described elsewhere [19]. Nevertheless, the main features are described below.

The displacing liquid is pumped by the use of a precise syringe pump ISCO-250D which allows a very accurate and stable flux without the need of any dampening system. The operation mode consists in fixing a determined flow and then, waiting for the pressure to stabilize. Increasing the flow stepwise, the experimental pairs of data of flow as function of pressure are obtained. As already explained at each equilibrium pressure the pores of a given size are opened.

Evidently the successive pressure increments (more precisely flow increments) should lead to higher permeability values until the asymptotic permeability $\left(\mathrm{L}_{\text {total }}\right)$ is reached. This asymptotic permeability should correspond to that of the actual membrane for the displacing liquid once all the pores have been emptied from the wetting liquid. However, in practice, due to uncertainty of experimental data consecutive data points could lead to values of decreasing permeability. These permeabilities would be interpreted as newly wetted pores which is impossible, thus, the data conversion algorithm eliminates these decreasing permeability points.

\subsection{Wetting and displacing liquids and physical parameters}

The employed wetting liquid was the alcoholic phase of a two phase mixture of Milli-Q water and isobutanol of reagent grade $(1: 1 \mathrm{w} / \mathrm{w})$ while the displacing liquid corresponds to the aqueous phase. The mixture was prepared by mixing proper amounts of water and alcohol into a separator funnel, shaking it vigorously and settling it overnight. The separated alcohol-rich phase was drained off and used as the wetting liquid and the 
aqueous-rich phase was used as the displacing liquid. The resulting surface tension between both liquids is $1.9 \mathrm{mN} / \mathrm{m}$ and the viscosity of the displacing liquid is $8.9 \cdot 10^{-4} \mathrm{~Pa} \cdot \mathrm{s}$, [22].

The last parameter, which is needed to convert flux distributions into absolute pore number distributions, is the pore length (1) which is estimated as either the thickness of the active layer in asymmetric membranes or the membrane thickness for symmetric ones. As this value is unknown for the characterized membranes, a pore length of $5 \mu \mathrm{m}$ was assumed. This assumption only affects the value of $\mathrm{N}$, but note that the effect of such assumption is the same for both the traditional method and the proposed approach since, in any case, both actually calculate the ratio N/l. Of course, either technique could be used to evaluate the porosity if the length is known and vice versa.

\section{Results and discussion}

\subsection{Physical meaning of the model parameters}

The proposed analyzing strategy for LLDP results consists in fitting the actual results to an ideal membrane which has a log-normal distribution of pores characterized by three parameters (N, R and $\mathrm{S}$ ). The $\mathrm{R}$ and $\mathrm{S}$ parameters (from the log-normal distribution) define the position and shape of the pore number distribution. An increase in $\mathrm{R}$ leads to a shift to the right of the distribution (higher membrane pores) whereas an increase in $\mathrm{S}$ means a broadening of the curve which is more significant for the largest pore sizes. On the other hand, the $\mathrm{N}$ parameter (coming from the Hagen-Poiseuille equation), does not affect the shape and features of the pore number distribution, but it is related with the overall flow that passes through the membrane. In other words, the higher $\mathrm{R}$ and/or $\mathrm{S}$ are, the lower $\mathrm{N}$ is 
needed to achieve a certain permeate flux is for a given membrane pore number distribution (flux is proportional to fourth power of radius according to Hagen-Poiseuille).

\subsection{Fitting the model to the experimental results}

The fitting of $\mathrm{R}, \mathrm{S}$ and $\mathrm{N}$ parameters was carried out for four different membranes with very different MWCOs ranging from 5 to $300 \mathrm{kDa}$ (see Fig. 1). Despite the differences in flow and pressure among membranes, the model fits very well the obtained experimental results with correlation coefficients higher than 0.999 for all membranes. The obtained geometric standard deviations are very similar for most of the membranes (from 1.3 to 1.5). The mean radius increases, as expected, as the MWCO of the membranes increases, following the order HFK-328 (1.5 nm - $5 \mathrm{kDa})$, UP020 (2.0 nm - $20 \mathrm{kDa})$, M030 (2.9 nm $30 \mathrm{kDa})$ and M300 (10.2 nm - $300 \mathrm{kDa})$. The total pore number density fluctuates around $4 \cdot 10^{16}$ pore $/ \mathrm{m}^{2}$ for the 5,20 and $30 \mathrm{kDa}$ membranes, but for M300 which has a high mean radius and thus, a lower total pore number density $\left(1.3 \cdot 10^{15}\right)$ is needed to achieve the given flows.

Therefore, a theoretical membrane defined by the three $\mathrm{N}, \mathrm{R}$ and $\mathrm{S}$ parameters can successfully model the flow experimental results of a LLDP analysis for very different membranes.

\section{Figure 1}

From the fittings shown in Fig. 1, it is possible to determine the flux or permeability distribution. This distribution accounts for the contribution (in percentage) of each pore size class to the total permeability. Fig. 2 shows these distributions for the four studied membranes. In all cases, the agreement between permeability distributions (shown as bars), 
coming from direct application of Grabar-Nikitine algorithm to experimental data, and the fitted ones (solid lines) is remarkable.

\section{Figure 2}

\subsection{Pore number distribution. Model validation}

The pore number distribution as a function of the pore radius is represented for the experimental and the modeled membranes to compare the theoretical membrane properties to those of the real one (Fig. 3).

It can be observed that the pore distribution of the HFK-328 theoretical membrane (Fig. 3a) fits very well to the Grabar-Nikitine treated data. For the rest of the membranes (Fig. 3), the pore size distribution fitting is not perfect but satisfactory.

In any case, the agreement between traditionally treated and fitted points is not as good as that obtained for permeability distributions (Fig. 2).This was predictable since permeability distributions come directly from experimental data while pore number ones need a transport model inside the pores to be applied (Hagen-Poiseuille). This was one of the conclusions of the Charmme Network [23] and is nowadays generally accepted

The theoretical distribution accurately matches in the area of higher radii whereas for the lower ones, there is a lack of experimental points. In any case, the agreement for the bigger pores would lead to good enough estimations of MWCO.

Finally a couple of physical limitations of the experimental apparatus should be pointed out because they would explain the lack of accurate experimental information for the smallest pores. It is important to consider that small pores are opened at high pressures, which sometimes cannot be reached either because of the physical limitations of the equipment (maximum pressure) or because the experimental run is automatically stopped when 
software detects non increasing permeabilities. This last procedure (based on a certain tolerance limit introduced by the operator) will prematurely stop the experiment, under certain circumstances, closing abruptly the distribution. Moreover, membranes having very high permeabilities (a usual target for membrane manufacturers) could lead to exhausting the pump reservoir $(500 \mathrm{~mL})$ before ending the analysis.

\section{Figure 3}

\subsection{Permeability and cumulative permeability distribution}

The experimental and modelled permeability cumulative distribution as well as the actual permeability as a function of the pore radius is depicted in Fig. 4. The modelled cumulative distributions of the permeability (closed symbols) do not fit the discretized values as well as it was seen for the differential permeability distribution.

The differences lie in the previously observed lack of experimental data for the lowest radii (high pressures). This implies that the smallest pores are not taken into account to determine their contribution to the global membrane permeability at a given pressure (for a given radius) and so, the permeability cumulative distribution is underestimated when using the experimental results for low radii. This seems more obvious as mean radius (or the MWCO) of the membrane increases, Fig 4d.

Although the lack of experimental data always appears at low radii, it has a different origin depending on the membrane. For instance, there is no data at radii lower than $1.0 \mathrm{~nm}$ for the HFK-328 membrane, because the measuring equipment cannot exceed 50 bar, whereas for the M300 the lowest radius from which there is information is $9.0 \mathrm{~nm}$, as for lower pore sizes, higher pressures are needed, lead to decreasing permeabilities that were automatically discarded by the setup control software. The contribution of pores around $1 \mathrm{~nm}$ to the 
overall permeability for HFK-328 is much lower to that of the pores around $9.0 \mathrm{~nm}$ for the M300 membrane. Therefore, the effect is more pronounced as the MWCO increases.

Nevertheless, the differences found in the individual contribution of each pore size to the overall permeability are not relevant to determine its actual value (Fig. 4, open symbols).

\section{Figure 4}

\subsection{Advantages of the theoretical model}

Once the model was validated, it can be used to improve results in the characterization of any membrane. The most important feature of this model is that it can be applied with a small amount of experimental data, allowing the extrapolation of the membrane performance throughout the LLDP analysis. This is of great importance, especially in quality control areas where saving time and costs are linked and a comprehensive and deep characterization should have done previously. Moreover, this model can also be useful to characterize many membranes in a short period of time, easing the membrane screening for a given process.

There are several parameters, such as the asymptotic permeability, the mean radius (based on either the pore number or the permeability) and MWCO estimation, which are usually employed to characterize membranes by LLDP. These parameters together with the fitted $\mathrm{R}, \mathrm{S}$ and $\mathrm{N}$ are gathered in Table 2 for the four tested membranes using different number of experimental data. For each studied membrane, the first two rows compare the resulting LLDP parameters obtained from direct application of Grabar-Nikitine algorithm with all the experimental data (row $\mathrm{GN}$ ) with the same parameters obtained by applying the previously explained model/technique (row M). For most studied parameters (asymptotic permeability and mean pore radius by permeability and number) modeled results are 
accompanied by an error estimation $(*)$ which considers values from the Grabar-Nikitine algorithm as the exact ones. In the case of MWCO, it makes no sense to compare modeled and experimental values as far as we have the nominal values of MWCO given by the membrane manufacturers. So in this case, error (**) accounts for differences between experimental and nominal values.

The information coming from manufacturers was considered reliable, despite they do not specifically state how cut-off was determined [17].

It is also interesting to test if the model still works well when only a small set of the experimental data is used in the fitting. In this sense, what has been done is to fit the developed model to a reduced number of data. In all cases it has been considered a minimum number of six experimental points (except for the last one, ItF, in which only three points were used), but the differences rely in which part of the experimental curve are those data pairs obtained from. Therefore, row In presents the results of the model applied using only the six pairs of flux, pressure data corresponding to the initial part of the experiment (corresponding to lower pressures). Next row (It) shows the fitting results using only six data pairs acquired at the intermediate section of the experiment. In next row (IF), the model was applied using 3 data pairs from the initial part together with the last three pairs. Finally, the last procedure accounts for using one data pair at the beginning of the experiment, another one at intermediate pressure values and last one from the end of the experiment(row ItF).

Finally, for all these partial procedures (In, It, IF, ItF), the error presented (***) has been calculated comparing the values of all the selected parameters with those values obtained from the model fitting using all data points (row M). 
The asymptotic permeability obtained by the model using all the experimental results (tag M) is similar to that experimentally determined for all the membranes, having relative errors lower than $6 \%$. Differences appear when the fittings are conducted using the results at low (tag In) and intermediate (tag It) pressures (flows), leading to differences around 10$20 \%$, except in the case of the M300 membrane, for which the fitting was not really possible in these conditions since errors are above $400 \%$. Nevertheless, when the fittings are conducted either with the data from the beginning (low pressure) and the end (high pressure) of the tests (tag IF) or with three distributed pairs of points (tag ItF) the modelled asymptotic permeability is again similar to the experimental one (tag GN) and to that modelled with all the experimental information (tag M). In fact, differences are lower than $5 \%$ for all the membranes.

Larger differences are found when the mean pore radius based on the pore number and on the permeability are analyzed. As it was seen in Fig. 3, there is a lack of experimental data of the pore distribution at low pore radius. Moreover, the permeability cumulative distribution is greatly affected as seen in Fig. 4. Therefore, there are high discrepancies between the mean radii values (based on both the pore number and the permeability) from the Grabar-Nikitine algorithm (tag GN) and those from the theoretical model (tag M), which range from 9 to $42 \%$. However, it is hard to establish which one of both is closer to the actual values for the membrane due to the lack of experimental information for low radii. In general, the mean pore radii (based on either the pore number or the permeability) obtained through the (In) and (It) procedures differ more from the values obtained using the all-data fitting (procedure M) than those using the (IF) and (ItF) procedures. Nonetheless, differences are small in general.. The fitting procedures give very good results, especially for the HFK-328 membrane. The ranges of the mean pore radius (based on the pore 
number) are relatively small for all the membranes: $1.1-1.6 \mathrm{~nm}, 1.2-2.0 \mathrm{~nm}, 2.2-2.6 \mathrm{~nm}$ and 8.3-11.3 nm for the HFK-328, UP020, M030 and M300 membranes, respectively. And a similar observation can be done for the mean pore radius based on permeability. Therefore, the possibility of using fewer experimental data to estimate the mean pore radius of the membranes using the proposed model is feasible.

The estimation of the MWCO leads to different conclusions depending on the membranes. The estimation of the HFK-328 and M300 membrane MWCOs is more accurate using the model (tag M) than using the Grabar-Nikitine algorithm results (GN), while for the UP020 and M030 membranes the model only allows an estimate of the order of magnitude of MWCO.

One of the shortcomings of applying this model to small amounts of experimental data is that the results could be incoherent. For instance, using the procedures (In) and (It) for very open UF membranes, such as the M300 membrane, the results are not successful because there is a lack of information in the high pressure area which is associated to the asymptotic permeability. Therefore, in these cases, a careful analysis of the results should be carried out, leading the LLDP experiment to include more high pressure points to avoid such errors.

\section{Table 2}

\section{Conclusions}

In this work a new approach to fit results from LLDP analysis has been proposed. Instead of the usual polynomial fitting equations without physical meaning, a log-normal pore size distribution was assumed for the membrane, requiring only three fitting parameters. 
Comparing the expected behavior of the model with the actual results, the log-normally shaped distribution is a reasonable choice.

The accuracy of the fitting approach has been tested with several polymeric membranes having an expected large range of pores, according to the nominal MWCO values. Results of the fitting are reasonably good and in accordance with most of the parameters arising from traditional LLDP analysis.

The use of partial data does not guarantee good results or show a clear trend in data analysis, in any case this partial analysis do not lead to worse results.

It must not be forgotten that a good fitting is only possible if good data is collected. Whatever is the procedure for measuring or fitting data, LLDP experiments are not and they will never be so easy to perform as GLDP ones, where only $5 \mathrm{~min}$ are enough to get a good pore size distribution.

On the contrary LLDP experiments need longer time (never less than $1 \mathrm{~h}$ ), so any approach aimed to reduce the number of experimental points, such as that considered in this work, requires a reliable data fitting for a possible extensive or commercial use of the technique.

\section{Acknowledgements}

Authors gratefully acknowledge the financial support given by: Junta de Castilla y León (Project VA248U13), Spanish Ministry of Science and Innovation (projects MAT201125513 and CTQ2012-31076) and the Spanish Ministry of Education, Culture and Sports via a collaboration scholarship and a FPU grant (AP2010-3549).

\section{Nomenclature}

A: Membrane surface area $\left(\mathrm{m}^{2}\right)$ 
$D_{\infty}$ : Diffusion coefficient of the dextran at infinite dilution in water $\left(\mathrm{m}^{2} / \mathrm{s}\right)$

$e_{k}$ : Difference between the experimental and the modelled membrane flow $\left(\mathrm{m}^{3} / \mathrm{s}\right)$

F: Membrane flux $\left(\mathrm{m}^{3} / \mathrm{m}^{2} \mathrm{~s}\right)$

$f_{L}(r)$ : Probability distribution function for the permeability from pores of radius $r\left(m^{-1}\right)$

$f_{n}(r)$ : Probability distribution function for the number of pores of radius $r\left(m^{-1}\right)$

J: Membrane flow $\left(\mathrm{m}^{3} / \mathrm{s}\right)$

1: Pore length

MWCO: Molecular weight cut-off (Da)

$\mathrm{n}(\mathrm{r})$ : Pore number distribution (pore $/ \mathrm{m}^{3}$ )

$\mathrm{N}$ : Total pore number density $\left(\right.$ pore $\left./ \mathrm{m}^{2}\right)$

$\mathrm{N}_{\mathrm{AB}}$ : Pore number density with radii between $\mathrm{r}_{\mathrm{A}}$ and $\mathrm{r}_{\mathrm{B}}\left(\right.$ pore $\left./ \mathrm{m}^{2}\right)$

$\Delta \mathrm{P}:$ Transmembrane pressure $(\mathrm{Pa})$

$\mathrm{R}$ : Geometric mean radius or location parameter (m)

r: radius $(\mathrm{m})$

$<\mathrm{r}\rangle_{\text {number: }}$ : Mean radius based on the pore number distribution (m)

$<\mathrm{r}\rangle_{\text {perm: }}$ Mean radius based on the permeability distribution (m)

S: Geometric standard deviation or scale parameter (dimensionless)

Greek letters

$\eta$ : Viscosity (Pa·s)

$\gamma:$ Interfacial tension $(\mathrm{N} / \mathrm{m})$ 


\section{References}

[1] D. Li, M.W. Frey, Y.L. Joo, Characterization of nanofibrous membranes with capillary flow porometry, Journal of Membrane Science, 286 (2006) 104-114.

[2] S. S. Manickam, J.R. McCutcheon, Characterization of polymeric nonwovens using porosimetry, porometry and X-ray computed tomography, Journal of Membrane Science, 407-408 (2012) 108-115.

[3] S. Munari, A. Bottino, G.C. Roda, G. Capannelli, Preparation of ultrafiltration membranes. State of the art, Desalination, 77 (1990) 85-100.

[4] P. Abaticchio, A. Bottino, G.C. Roda, G. Capannelli, S. Munari, Characterization of ultrafiltration polymeric membranes, Desalination, 78 (1990) 235-255.

[5] J.I. Calvo, A. Bottino, P. Prádanos, L. Palacio, A. Hernández, Porosity, in: E.M.V. Hoek, V.V. Tarabara (Eds.) Encyclopedia of Membrane Science and Technology, John Wiley and Sons, Hoboken, NJ (USA), 2014.

[6] A. Hernández, J.I. Calvo, P. Prádanos, F. Tejerina, Pore size distributions in microporous membranes. A critical analysis of the bubble point extended method, Journal of Membrane Science, 112 (1996) 1-12.

[7] K.R. Morison, A comparison of liquid-liquid porosimetry equations for evaluation of pore size distribution, Journal of Membrane Science, 325 (2008) 301-310.

[8] T.N. Shah, H.C. Foley, A.L. Zydney, Development and characterization of nanoporous carbon membranes for protein ultrafiltration, Journal of Membrane Science, 295 (2007) 4049.

[9] S. Munari, A. Bottino, P. Moretti, G. Capannelli, I. Becchi, Permoporometric study on ultrafiltration membranes, Journal of Membrane Science, 41 (1989) 69-86. 
[10] P. Aimar, M. Meireles, V. Sanchez, A contribution to the translation of retention curves into pore size distributions for sieving membranes, Journal of Membrane Science, 54 (1990) 321-338.

[11] K.D. Knierim, M. Waldman, E.A. Mason, Bounds on solute flux and pore-size distributions for non-sieving membranes, Journal of Membrane Science, 17 (1984) 173203.

[12] K.D. Knierim, E.A. Mason, Heteroporous sieving membranes: Rigorous bounds on pore-size distributions and sieving curves, Journal of Membrane Science, 42 (1989) 87-107. [13] A.L. Zydney, P. Aimar, M. Meireles, J.M. Pimbley, G. Belfort, Use of the log-normal probability density function to analyze membrane pore size distributions: functional forms and discrepancies, Journal of Membrane Science, 91 (1994) 293-298.

[14] P. Grabar, S. Nikitine, Sur le diamètre des pores des membranes en collodion utilisées en ultrafiltration, Journal of Chemical Physics, 33 (1936) 721-741.

[15] F. Erbe, The determination of pore distributions according to sizes in filters and ultrafilters, Kolloid-Z, 63 (1933) 277-285.

[16] S. Derjani-Bayeh, V.G.J. Rodgers, Sieving variations due to the choice in pore size distribution model, Journal of Membrane Science, 209 (2002) 1-17.

[17] J.I. Calvo, R.I. Peinador, P. Prádanos, L. Palacio, A. Bottino, G. Capannelli, A. Hernández, Liquid-liquid displacement porometry to estimate the molecular weight cut-off of ultrafiltration membranes, Desalination, 268 (2011) 174-181.

[18] P. Carretero, S. Molina, A. Lozano, J. de Abajo, J.I. Calvo, P. Prádanos, L. Palacio, A. Hernández, Liquid-liquid displacement porosimetry applied to several MF and UF membranes, Desalination, 327 (2013) 14-23. 
[19] J.M. Sanz, R. Peinador, J.I. Calvo, A. Hernández, A. Bottino, G. Capannelli, Characterization of UF membranes by liquid-liquid displacement porosimetry, Desalination, 245 (2009) 546-553.

[20] M.C. Almécija, J.E. Zapata, A. Martinez-Ferez, A. Guadix, A. Hernández, J.I. Calvo, E.M. Guadix, Analysis of cleaning protocols in ceramic membranes by liquid-liquid displacement porosimetry, Desalination, 245 (2009) 541-545.

[21] J.I. Calvo, A. Bottino, G. Capannelli, A. Hernández, Pore size distribution of ceramic UF membranes by liquid-liquid displacement porosimetry, Journal of Membrane Science, 310 (2008) 531-538.

[22] J.I. Calvo, A. Bottino, G. Capannelli, A. Hernández, Comparison of liquid-liquid displacement porosimetry and scanning electron microscopy image analysis to characterise ultrafiltration track-etched membranes, Journal of Membrane Science, 239 (2004) 189-197. [23] CHARMME Network "Harmonization of characterization methodologies for porous membranes", EC Contract SMT4-CT 98-7518. Web page: http://www.charmme.livstek.lth.se. 


\section{Figures}


Figure 1
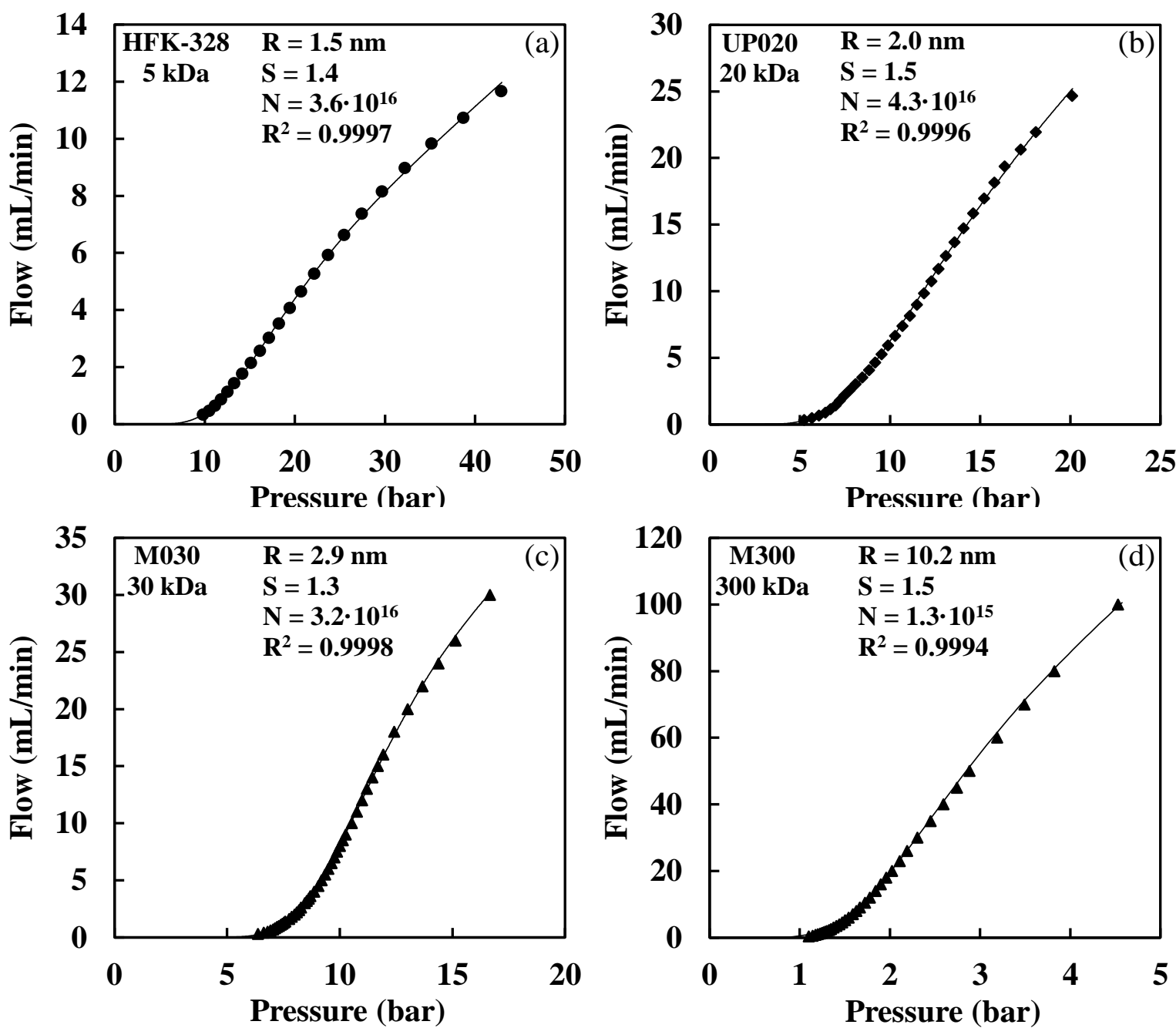

Fig. 1. Experimental results obtained through LLDP (symbols) and fitted model (solid line) for HFK-328 (a), UP020 (b), M030 (c), and M300 (d) membranes. 
Figure 2
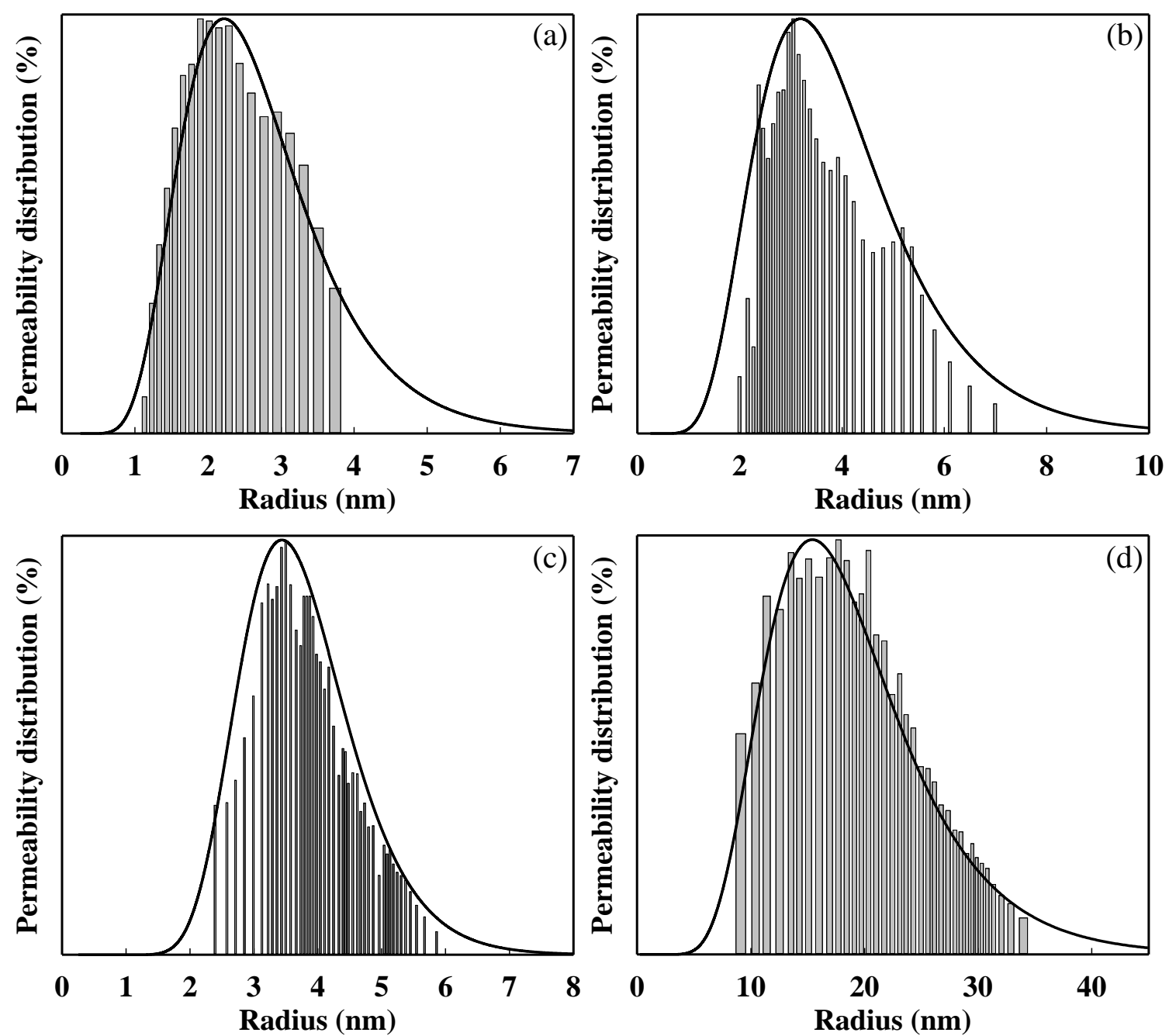

Fig. 2. Experimental (bars) and modeled (solid line) permeability distribution of HFK328 (a), UP020 (b), M030 (c) and M300 (d) membranes. 
Figure 3
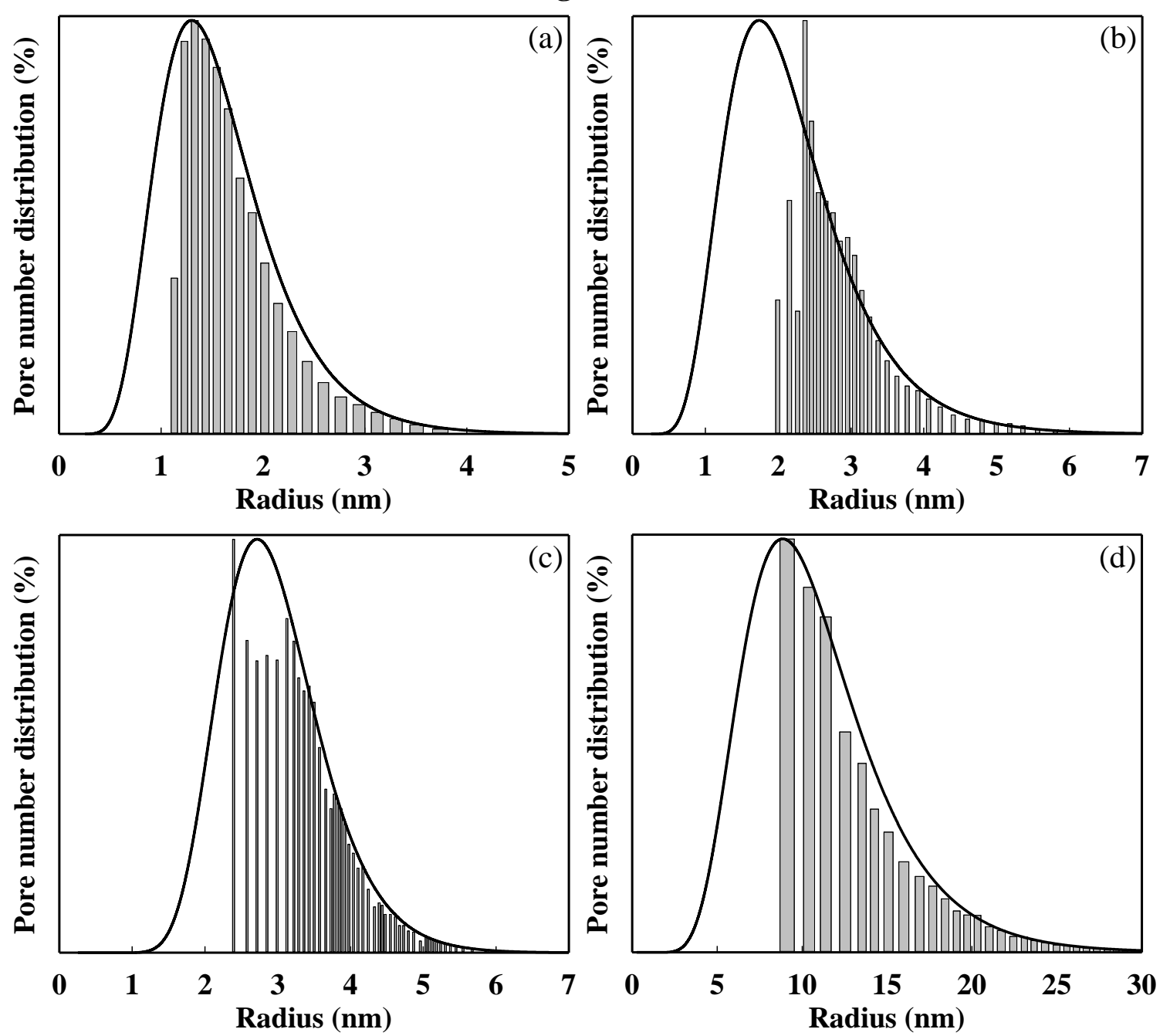

Fig. 3. Experimental (bars) and modelled (solid line) pore number distribution of HFK-328 (a), UP020 (b), M030 (c) and M300 (d) membranes. 


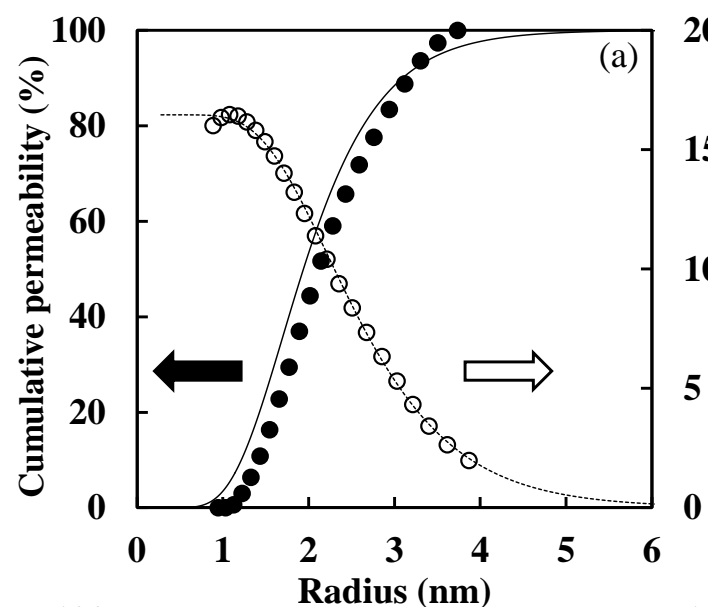

Figure 4
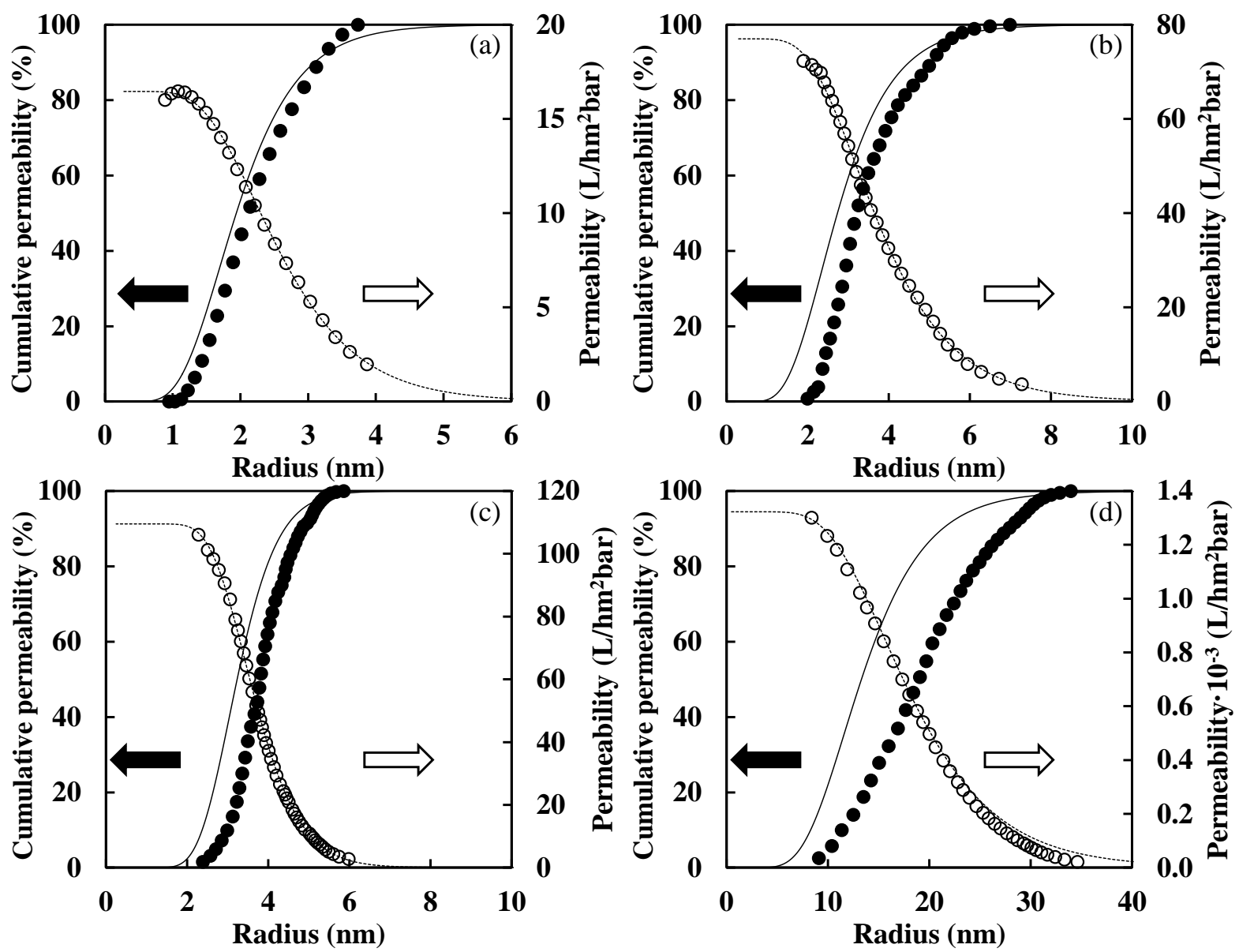

Fig. 4. Experimental (closed symbols) and modelled (solid line) cumulative permeability distribution as well as experimental (open symbols) and modelled (dotted line) permeability as function of pore size for the HFK-328 (a), UP020 (b), M030 (c) and M300 (d) membranes. 


\section{Tables}


Table 1: Membrane properties according to manufacturers.

\begin{tabular}{cccc}
\hline Membrane & Material & Manufacturer & MWCO (kDa) \\
\hline HFK-328 & Polyethersulfone & Koch & 5 \\
UP020 & & Nadir & 20 \\
Minitan M030 & Polysulfone & Millipore & 30 \\
Minitan M300 & & & 300 \\
\hline
\end{tabular}


Table 2: Main membrane parameters obtained through LLDP.

\begin{tabular}{|c|c|c|c|c|c|c|c|c|c|c|c|c|}
\hline Membr. & $\begin{array}{l}\text { Math. } \\
\text { proced. }\end{array}$ & $\begin{array}{c}\text { Asymptotic } \\
\text { permeability } \\
\left(\mathrm{L} / \mathrm{hm}^{2} \text { bar }\right)\end{array}$ & $\begin{array}{c}\text { Relative } \\
\text { error }(\%) \\
(*-* * *)\end{array}$ & $\begin{array}{l}\text { Mean radius - } \\
\text { Pore number } \\
(\mathbf{n m})\end{array}$ & $\begin{array}{c}\text { Relative } \\
\text { error }(\%) \\
(* \ldots * *)\end{array}$ & $\begin{array}{c}\text { Mean radius - } \\
\text { Permeability } \\
(\mathbf{n m})\end{array}$ & $\begin{array}{c}\text { Relative } \\
\text { error }(\%) \\
\left(* *_{-} * * *\right)\end{array}$ & $\begin{array}{c}\text { MWCO } \\
\text { (kDa) }\end{array}$ & $\begin{array}{c}\text { Relative } \\
\text { error }(\%) \\
(* *)\end{array}$ & $\begin{array}{c}\mathbf{R} \\
(\mathbf{n m})\end{array}$ & $\mathbf{S}$ & $\mathbf{N}$ \\
\hline \multirow{6}{*}{$\begin{array}{c}\text { HFK- } \\
328\end{array}$} & GN & 16.5 & - & 1.7 & - & 2.3 & - & 6.9 & 38 & - & - & - \\
\hline & $\mathrm{M}$ & 16.5 & $1^{*}$ & 1.2 & $27^{*}$ & 2.1 & $9^{*}$ & 4.7 & 6 & 1.5 & 1.44 & $3.7 \cdot 10^{16}$ \\
\hline & In & 17.0 & $3^{* * * *}$ & 1.3 & $8^{* * *}$ & 2.1 & $3^{* * *}$ & 5.3 & 7 & 1.6 & 1.42 & $3.3 \cdot 10^{16}$ \\
\hline & It & 17.0 & $3^{* * *}$ & 1.1 & $13^{* * *}$ & 2.0 & $5^{* * *}$ & 3.7 & 26 & 1.3 & 1.48 & $5.0 \cdot 10^{16}$ \\
\hline & IF & 16.3 & $1^{* * *}$ & 1.6 & $28^{* * * *}$ & 2.3 & $11^{* * * *}$ & 7.2 & 43 & 1.8 & 1.37 & $2.2 \cdot 10^{16}$ \\
\hline & $\mathrm{ItF}$ & 16.1 & $2^{* * * *}$ & 1.2 & $1^{* * *}$ & 2.1 & $1^{* * *}$ & 4.8 & 3 & 1.5 & 1.44 & $3.5 \cdot 10^{16}$ \\
\hline \multirow{6}{*}{ UP020 } & $\mathrm{GN}$ & 72 & - & 2.8 & - & 3.5 & - & 18 & 8 & - & - & - \\
\hline & $\mathrm{M}$ & 77 & $6^{*}$ & 1.6 & $42^{*}$ & 3.0 & $17^{*}$ & 9 & 56 & 2.0 & 1.48 & $4.3 \cdot 10^{16}$ \\
\hline & In & 87 & $12^{* * *}$ & 1.2 & $28^{\text {*** }}$ & 2.5 & $15^{* * *}$ & 5 & 75 & 1.6 & 1.55 & $1.0 \cdot 10^{17}$ \\
\hline & It & 68 & $12^{* * *}$ & 1.9 & $17^{* * *}$ & 3.2 & $9^{* * *}$ & 12 & 41 & 2.3 & 1.44 & $2.7 \cdot 10^{16}$ \\
\hline & IF & 74 & $4^{* * *}$ & 2.0 & $24^{* * *}$ & 3.3 & $11^{* * *}$ & 13 & 35 & 2.4 & 1.42 & $2.6 \cdot 10^{16}$ \\
\hline & $\mathrm{ItF}$ & 76 & $1^{* * * *}$ & 1.5 & $9^{* * *}$ & 2.9 & $2^{* * *}$ & 8 & 62 & 1.9 & 1.51 & $4.9 \cdot 10^{16}$ \\
\hline \multirow{6}{*}{ M030 } & GN & 106 & - & 3.4 & - & 3.9 & - & 27 & 9 & - & - & - \\
\hline & $\mathrm{M}$ & 110 & $3^{*}$ & 2.6 & $22^{*}$ & 3.3 & $14^{*}$ & 19 & 38 & 2.9 & 1.27 & $3.2 \cdot 10^{16}$ \\
\hline & In & 116 & $6^{* * *}$ & 2.2 & $15^{* * *}$ & 3.0 & $9^{* * *}$ & 14 & 53 & 2.5 & 1.32 & $5.0 \cdot 10^{16}$ \\
\hline & It & 123 & $13^{* * * *}$ & 2.3 & $13^{* * *}$ & 3.1 & $7^{* * *}$ & 15 & 51 & 2.6 & 1.32 & $5.0 \cdot 10^{16}$ \\
\hline & IF & 110 & $1^{* * *}$ & 2.5 & $5^{* * *}$ & 3.3 & $2^{* * *}$ & 17 & 43 & 2.8 & 1.29 & $3.5 \cdot 10^{16}$ \\
\hline & $\mathrm{ItF}$ & 112 & $2^{* * *}$ & 2.4 & $8^{* * * *}$ & 3.2 & $4^{* * *}$ & 16 & 46 & 2.7 & 1.30 & $3.9 \cdot 10^{16}$ \\
\hline \multirow{6}{*}{ M300 } & GN & 1302 & - & 13.2 & - & 19.6 & - & 629 & 110 & - & - & - \\
\hline & $\mathrm{M}$ & 1322 & $2^{*}$ & 8.3 & $37^{*}$ & 14.4 & $27^{*}$ & 261 & 13 & 10.2 & 1.45 & $1.3 \cdot 10^{15}$ \\
\hline & In & 10365 & $700^{* * *}$ & 5.5 & $34^{* * *}$ & 9.7 & $33^{* * *}$ & 111 & 63 & 6.8 & 1.46 & $5.0 \cdot 10^{16}$ \\
\hline & It & 6160 & $400^{* * *}$ & 4.5 & $46^{* * *}$ & 9.0 & $38^{* * *}$ & 78 & 74 & 5.8 & 1.52 & $4.3 \cdot 10^{16}$ \\
\hline & $\mathrm{IF}$ & 1334 & $1^{* * *}$ & 8.6 & $5^{* * *}$ & 14.0 & $3^{* * *}$ & 271 & 10 & 10.3 & 1.41 & $1.4 \cdot 10^{15}$ \\
\hline & $\mathrm{ItF}$ & 1308 & $1^{* * * *}$ & 11.3 & $37^{* * *}$ & 16.1 & $12^{* * *}$ & 430 & 43 & 12.9 & 1.35 & $7.4 \cdot 10^{14}$ \\
\hline
\end{tabular}

GN: Experimental results

M: Fitted with all experimental data

In: Fitted with the first six pairs of data

It: Fitted with six pairs of data at intermediate fluxes

IF: Fitted with the first and last three pairs of data

ItF: Fitted with the first one, one at the middle and the last one pairs of data 